\title{
STAR Results from the RHIC Beam Energy Scan-I
}

\author{
Lokesh Kumar (for the STAR Collaboration) ${ }^{1}$ \\ Department of Physics, Kent State University, Kent, $\mathrm{OH} 44242$
}

\begin{abstract}
The Beam Energy Scan (BES) program is being pursued at RHIC to study the QCD phase diagram, and search for the possible QCD phase boundary and possible QCD critical point. The data for Phase-I of the BES program have been collected for Au+Au collisions at center-of-mass energies $\left(\sqrt{s_{N N}}\right)$ of 7.7, 11.5, 19.6, 27, and $39 \mathrm{GeV}$. These collision energies allowed the STAR experiment to cover a wide range of baryon chemical potential $\mu_{B}(100-400 \mathrm{MeV})$ in the QCD phase diagram. We report on several interesting results from the BES Phase-I covering the high net-baryon density region. These results shed light on particle production mechanism and freezeout conditions, first-order phase transition and "turn-off" of QGP signatures, and existence of a critical point in the phase diagram. Finally, we give an outlook for the future BES Phase-II program and a possible fixed target program at STAR.
\end{abstract}

\section{Introduction}

The results from top RHIC energies suggest the existence of the Quark Gluon Plasma (QGP) [1]. The main task at hand now is to study the properties of the QGP and establish the QCD phase diagram. Lattice QCD calculations predict the transition between QGP and the hadronic gas as crossover at $\mu_{B}=0$, while at large $\mu_{B}$ they predict a first order phase transition [2]. A point where the first order phase transition ends is called the critical point. Experimentally, the QCD phase diagram can be studied by colliding heavy ions at varying beam energies that can provide a $T-\mu_{B}$ region for each energy. Then, one can look at the various signatures for the phase boundary and critical point. We present results on (a) freeze-out parameters to get insight into the QCD phase diagram, (b) first and second coefficients $\left(v_{1}, v_{2}\right)$ of the Fourier expansion of the angular distributions, dynamical charge correlations, and nuclear modification factor $R_{\mathrm{CP}}$ for the search of first-order phase transition and "turn-off" of QGP signatures, and (c) fluctuation measurements for the search of QCD critical point.

The first STAR proposal for the BES program [3] was made in the year 2008. This was followed by a successful data taking and physics analysis of a test run below injection energies at $\sqrt{s_{N N}}=9.2 \mathrm{GeV}[4]$. The first phase of the BES program was started in the year 2010 with data taking at three low energies of 7.7, 11.5, and $39 \mathrm{GeV}$. In the year 2011, two more energy points were added at $\sqrt{s_{N N}}=19.6$ and $27 \mathrm{GeV}$.

\footnotetext{
${ }^{1}$ A list of members of the STAR Collaboration and acknowledgements can be found at the end of this issue.
} 
The results presented here are based on the data collected by the STAR detector. STAR covers a large acceptance of $2 \pi$ in azimuth $(\phi)$ and -1 to 1 in pseudorapidity $(\eta)$. One of the important advantages which STAR possesses for the BES program is its almost uniform acceptance for different identified particles and collision energies at midrapidity. The main tracking device at STAR is the Time Projection Chamber (TPC), which provides momentum as well as particle identification (PID). For higher transverse momentum $\left(p_{T}\right)$ region, the Time Of Flight (TOF) detector is quite effective in distinguishing different particles. Particles are identified using the ionization energy loss in TPC and time-of-flight information from TOF [5]. The centrality selection is done in STAR using the uncorrected charge particle multiplicity measured in the TPC within $|\eta|<0.5[4]$.

\section{Accessing QCD Phase Diagram}

The QCD phase diagram is the variation of temperature $T$ and baryon chemical potential $\mu_{B}$. These quantities can be extracted from the measured hadron yields. Transverse momentum spectra for the BES Phase-I energies are obtained for $\pi, K, p, \Lambda, \Xi, K_{S}^{0}$, and $\phi[6]$. The particle ratios are used to obtain the chemical freeze-out (a state when the yields of particles get fixed) conditions using the statistical thermal model (THERMUS) [7]. The two main extracted parameters are chemical freeze-out temperature $T_{\mathrm{ch}}$ and $\mu_{B}$. Figure1 (left panel) shows the variation of the extracted chemical freeze-out parameters using the Grand-Canonical Ensemble (GCE) approach of THERMUS for different energies and centralities [8]. We observe that at lower energies, $T_{\mathrm{ch}}$ shows a variation with $\mu_{B}$ as a function of centrality. Both $T_{\mathrm{ch}}$ and $\mu_{B}$ values seem to be decreasing from central to peripheral collisions. It may be noted that use of Strangeness Canonical Ensemble (SCE) shows an opposite trend, i.e. the $T_{\text {ch }}$ values seem to increase from central to peripheral collisions [8]. However, it is found that the $\chi^{2} / N D F$ in case of SCE is higher for peripheral collisions [8]. More investigations are ongoing. In GCE the energy and quantum numbers or particle numbers are conserved on average through the temperature and chemical potentials. GCE is widely used in heavy-ion collisions. In the SCE, the strangeness $(S)$ in the system is fixed exactly by its initial value of $S$, while the baryon and charge contents are treated grand-canonically. Although the $T_{\mathrm{ch}}$ shows an opposite trend between GCE and SCE, both results suggest the variation of chemical freeze-out parameters with centrality at lower energies.

The particle spectra can be used to obtain the kinetic freeze-out (a state when the spectral shapes of particles get fixed) conditions using the Blast Wave (BW) model [11]. The BW model is used to simultaneously fit the $\pi, K, p$ spectra and the two relevant extracted parameters are kinetic freeze-out temperature $T_{\text {kin }}$ and average flow velocity $\langle\beta\rangle$. Figure1 (right panel) shows the variation of kinetic freeze-out parameters for different energies and centralities [8]. We observe that at a given collision energy, there is an anti-correlation between $T_{\text {kin }}$ and $\langle\beta\rangle$. For a given collision centrality, the freeze-out temperature at high energy is lower and the average collectivity velocity $\langle\beta\rangle$ is larger due to expansion.

\section{Search for First Order Phase Transition \& Turn-off of QGP Signatures}

\subsection{Directed Flow}

The directed flow $v_{1}$ is calculated as $\left\langle\cos \left(\phi-\Psi_{1}\right)\right\rangle$, where $\phi$ and $\Psi_{1}$ are the azimuthal angle of the produced particles and orientation of the first-order event plane, respectively. The directed 

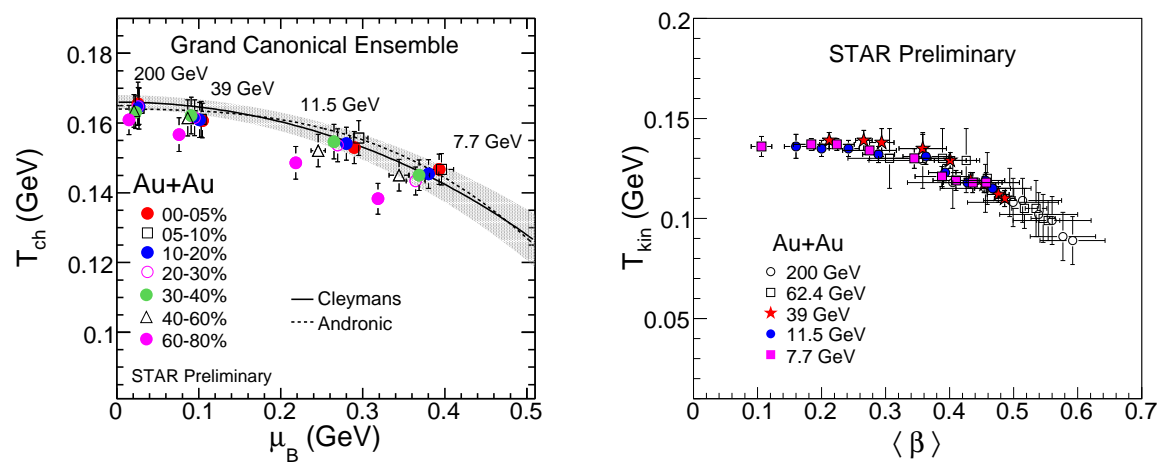

Figure 1: (Color online) Left panel: Variation of $T_{\mathrm{ch}}$ with $\mu_{B}$ for different energies and centralities. The curves represent the theoretical calculations [9, 10]. Right panel: Variation of $T_{\text {kin }}$ with $\langle\beta\rangle$ for different energies and centralities. Errors in both panels represent the quadrature sum of systematic and statistical errors.

flow measurements near midrapidity for protons are considered as sensitive to the equation of state (EOS) and hence can be considered a phase transition signal [12]. Figure 2] shows the $v_{1}$

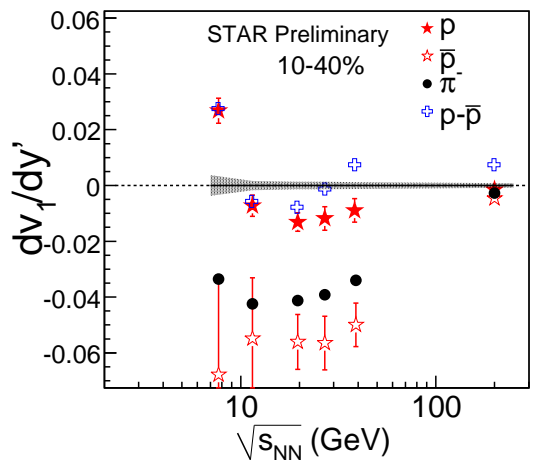

Figure 2: (Color online) Directed flow slope $\left(d v_{1} / d y^{\prime}, y^{\prime}=y / y_{\text {beam }}\right)$ for $\pi^{-}, p, \bar{p}$, and netprotons $(p-\bar{p})$ near midrapidity as a function of beam energy for mid-central (10-40\%) $\mathrm{Au}+\mathrm{Au}$ collisions. The shaded band refers to the systematic uncertainty on net-proton measurements.

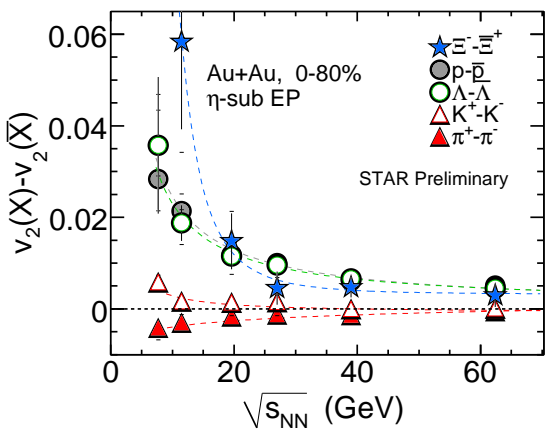

Figure 3: (Color online) The difference in $v_{2}$ between particles and their corresponding anti-particles as a function of beam energy in $0-80 \% \mathrm{Au}+\mathrm{Au}$ collisions. The curves represent fits to data points as discussed in text. Both statistical (vertical lines) and systematic errors (caps) are shown.

slope $\left(d v_{1} / d y^{\prime}\right.$, where $y^{\prime}=y / y_{\text {beam }}$ and $y$ is rapidity), near midrapidity as a function of beam energy for the mid-central (10-40\%) Au+Au collisions [13]. The pion and anti-proton $v_{1}$ slopes show negative values for all the beam energies studied. The proton $v_{1}$ slope changes sign while going from $7.7 \mathrm{GeV}$ to $11.5 \mathrm{GeV}$ and then stays negative up to $200 \mathrm{GeV}$. However, the netprotons $v_{1}$ slope (obtained using $v_{1}$ slopes of $p, \bar{p}$ and ratio of $\bar{p} / p$ ) changes sign two times going from lower to higher energy while showing a dip around $\sqrt{s_{N N}}=10-20 \mathrm{GeV}$. More studies are needed in order to understand these interesting observations. 


\subsection{Elliptic Flow}

The elliptic flow $v_{2}$ is calculated as $\left\langle\cos 2\left(\phi-\Psi_{2}\right)\right\rangle$, where $\Psi_{2}$ is orientation of the secondorder event plane. Elliptic flow mainly probes the early stages of heavy-ion collisions. At top RHIC energy of $200 \mathrm{GeV}$ in $\mathrm{Au}+\mathrm{Au}$ collisions, the elliptic flow scaled by the number of constituent quarks $\left(n_{q}\right)$ vs. $\left(m_{T}-m_{0}\right) / n_{q}$ (where $m_{T}=\sqrt{p_{T}^{2}+m_{0}^{2}}$ ) shows a scaling behavior where mesons and baryons have similar values at intermediate $p_{T}$. This is referred to as the number of constituent quark (NCQ) scaling [14]. It is an established signature of partonic matter formed in $\mathrm{Au}+\mathrm{Au}$ collisions at $200 \mathrm{GeV}$ and deviations from such scaling would indicate the dominance of hadronic interactions. Hence breaking of NCQ scaling at lower energies could be an indication of a "turn-off" of QGP signatures. Figure 3 shows the difference in $v_{2}$ of particles and

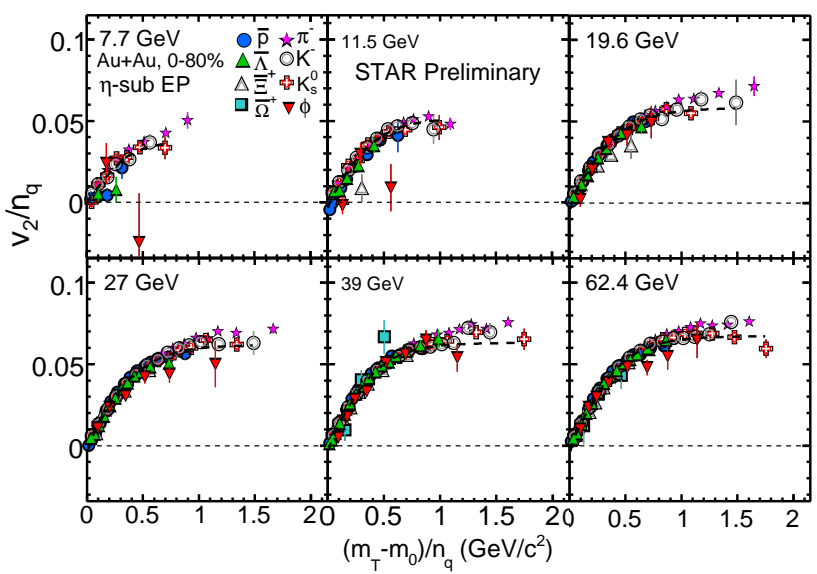

Figure 4: (Color online) $v_{2} / n_{q}$ as a function of $\left(m_{T}-m_{0}\right) / n_{q}$ for different particles in $\mathrm{Au}+\mathrm{Au}$ collisions at $\sqrt{s_{N N}}=7.7,11.5,19.6,27,39$ and $62.4 \mathrm{GeV}$. The errors shown are statistical only.

corresponding anti-particles as a function of beam energy [15]. The curves represent fits to data points with functional form: $f_{\Delta v_{2}}\left(\sqrt{s_{N N}}\right)=a \sqrt{s_{N N}}-b+c$. The $v_{2}$ difference between particles and anti-particles is observed to increase when we go towards the lower energies. At low energies, $v_{2}\left(\pi^{-}\right)>v_{2}\left(\pi^{+}\right), v_{2}\left(K^{+}\right)>v_{2}\left(K^{-}\right)$, and $v_{2}$ (baryons) $>v_{2}$ (anti-baryons). This difference between particles and anti-particles suggests that the NCQ scaling among particles and anti-particles is broken. However, the observed difference between $v_{2}$ of particles and anti-particles could be qualitatively explained by the models incorporating baryon transport at midrapidity and hadronic interactions [16]. We also observe that the baryons-mesons splitting for $v_{2}$ vs. $m_{T}-m_{0}$ starts to disappear for anti-particles at $11.5 \mathrm{GeV}$ and below. Figure 4 shows the $v_{2} / n_{q}$ vs. $\left(m_{T}-m_{0}\right) / n_{q}$ for different particles for $\sqrt{s_{N N}}=7.7-62.4 \mathrm{GeV}$ [15]. We observe that results for all the particles are consistent among each other within $\pm 10 \%$ level, except for the $\phi$-mesons. At the largest $m_{T}-m_{0}$ the $\phi$-meson data points deviate by $1.8 \sigma$ and $2.3 \sigma$ for $\sqrt{s_{N N}}=7.7$ and $11.5 \mathrm{GeV}$, respectively. Since $\phi$-mesons have smaller hadronic interaction cross-section, their smaller $v_{2}$ values could indicate that the hadronic interactions start to dominate over partonic effects for the systems formed at beam energies below $\sqrt{s_{N N}}=11.5 \mathrm{GeV}$ [17]. However, as can been seen from the figure, a higher statistics data are needed to extend the $m_{T}-m_{0}$ range and significance of the deviation observed. 


\subsection{Dynamical Charge Correlations}

The dynamical charge correlations are studied through a three-particle mixed harmonics azimuthal correlator [18], $\gamma=\left\langle\cos \left(\phi_{\alpha}+\phi_{\beta}-2 \Psi_{\mathrm{RP}}\right)\right\rangle$. This observable represents the difference between azimuthal correlations projected onto the direction of the angular momentum vector and correlations projected onto the collision reaction plane. It is suggested that the difference in the

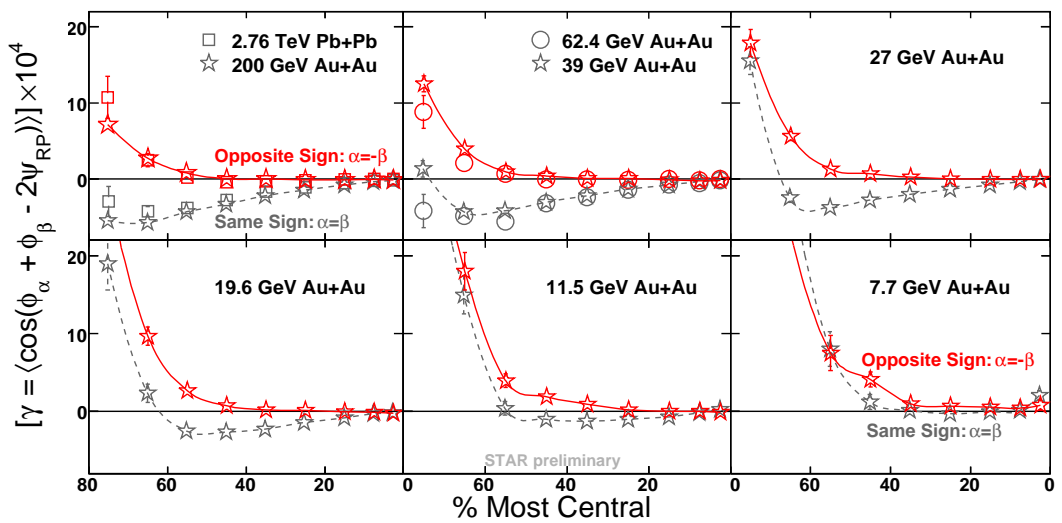

Figure 5: (Color online) $\left\langle\cos \left(\phi_{\alpha}+\phi_{\beta}-2 \Psi_{\mathrm{RP}}\right)\right\rangle$ as a function of centrality for Au+Au collisions at $\sqrt{s_{N N}}=7.7-200 \mathrm{GeV}$. For comparison, results for $\mathrm{Pb}+\mathrm{Pb}$ collisions at $2.76 \mathrm{TeV}$ are also shown. Errors are statistical only.

correlations between same sign and opposite sign charges in heavy-ion collisions could be related to local parity violation if there is a deconfinement and a chiral phase transition [19]. This is also referred to as Chiral Magnetic Effect (CME). At top RHIC energies, we observed a separation between the correlations of same and opposite sign charges. If this difference can be attributed to the QCD phase transitions, the absence of such observation could be an indication of the system which did not undergo the phase transition. Hence, the observable could be useful to locate the energy in the BES program where the QGP signature "turns off". Figure 5 shows the results for the beam energies from 7.7-200 GeV as a function of centrality [20]. For comparison, $\mathrm{Pb}-\mathrm{Pb}$ results from ALICE are also shown [21] which are observed to be consistent with the results from top RHIC energy. The separation between same and opposite sign charges decreases with decreasing energy and vanishes below $\sqrt{s_{N N}}=11.5 \mathrm{GeV}$.

\subsection{Nuclear Modification Factor}

Another established signature of QGP at top RHIC energy is the Nuclear Modification Factor $R_{\mathrm{CP}}$, which is defined as ratio of yields at central collisions to peripheral collisions, scaled by the corresponding number of binary collisions. It has been observed that at high $p_{T}$, the $R_{\mathrm{CP}}$ of different particles is less than unity [22], which is attributed to the energy loss of the partons in the dense medium. In the absence of dense medium, there may not be suppression of high $p_{T}$ particles, which can serve as an indication of "turn-off" of a QGP signature. Figure 6 shows the $R_{\mathrm{CP}}$ of various strange hadrons such as $K_{S}^{0}, \phi, \Lambda, \Xi^{-}$, and $\Omega^{-}$in Au+Au collisions at $\sqrt{s_{N N}}=7.7-$ $39 \mathrm{GeV}$ [23]. We observe that for $p_{T}>2 \mathrm{GeV} / c$, the $R_{\mathrm{CP}}\left(K_{S}^{0}\right)$ is less than unity at $39 \mathrm{GeV}$ and then the value increases as the beam energy decreases. For $\sqrt{s_{N N}}<19.6 \mathrm{GeV}, R_{\mathrm{CP}}\left(K_{S}^{0}\right)$ is above unity, indicating decreasing partonic effects at lower energies. For (un)-identified charged hadrons results, please refer to [24]. 


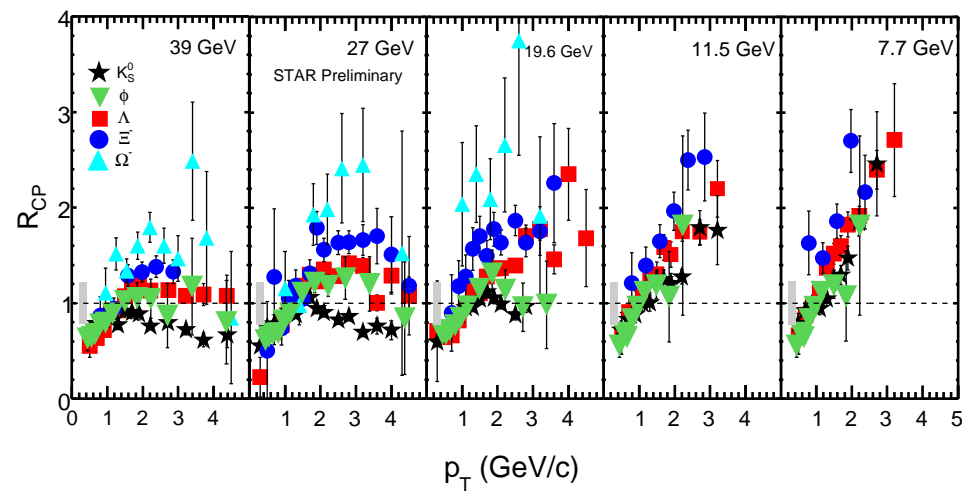

Figure 6: (Color online) $R_{\mathrm{CP}}(0-5 \% / 40 \%-60 \%)$ for $K_{S}^{0}, \Lambda, \Xi^{-}, \Omega^{-}$and $R_{\mathrm{CP}}(0-10 \% / 40 \%-$ $60 \%$ ) for $\phi$-meson in $\mathrm{Au}+\mathrm{Au}$ collisions at $\sqrt{s_{N N}}=7.7-39 \mathrm{GeV}$. Errors are statistical only. Grey bands represent the normalization error from $N_{\text {bin }}$.

\section{Search for QCD Critical Point}

If a system passes close to a critical point, the correlation length $\xi$ is expected to diverge. The higher moments (skewness $S$ and kurtosis $\kappa$ ) of distributions of conserved quantities such as net-baryons, net-charge, and net-strangeness, have a better sensitivity to the correlation length compared to variance $\sigma^{2}$ [25]. Also, the moment products such as $\kappa \sigma^{2}$ and $S \sigma$ can be related to the ratios of order susceptibilities calculated in Lattice QCD and HRG model as $\kappa \sigma^{2}=\chi_{B}^{(4)} / \chi_{B}^{(2)}$ and $S \sigma=\chi_{B}^{(3)} / \chi_{B}^{(2)}[26]$. One of the advantages of using these products is that they cancel the volume effects. Figure 7 shows the $\kappa \sigma^{2}$ and $S \sigma$ for net-protons as a function of beam energy for different collision centralities [27]. For comparison, the results are shown for Poisson expectations and UrQMD model calculations [28]. The bottom panel shows the $S \sigma$ values normalized by the corresponding Poisson expectations. We observe that the moment products show similar values for central 0-5\% and peripheral collisions for $\sqrt{s_{N N}}=39-200 \mathrm{GeV}$. For beam energies below $39 \mathrm{GeV}$, they have different values for central and peripheral collisions. Their values are below Poisson expectations for $\sqrt{s_{N N}}>7.7 \mathrm{GeV}$ for $0-5 \%$ central collisions. However, for peripheral collisions, moment products values are greater than Poisson expectations below 19.6 $\mathrm{GeV}$. The UrQMD model calculations show a smooth monotonic behavior as a function of collision energy. It may be noted that we have large uncertainties for data points below $19.6 \mathrm{GeV}$ that calls for higher statistics data for these energies. For net-charge and particle ratio fluctuation results, please refer to [29].

\section{The BES Phase-II and STAR in Fixed Target Mode}

The BES Phase-I program from STAR has several interesting results as discussed above. We have collected sizable data for $\mathrm{Au}+\mathrm{Au}$ collisions over a wide range of collisions energies from 7.7 to $39 \mathrm{GeV}$. However, to consolidate the findings from Phase-I, we need higher statistics at lower energies, especially at 7.7 and $11.5 \mathrm{GeV}$. As we have discussed above, statistics for several important observables, such as $\phi$-meson $v_{2}$ and higher moments of net-protons distributions, are not sufficient to draw quantitative conclusions. Also, there is more than a $100 \mathrm{MeV}$ gap in $\mu_{B}$ between 11.5 and $19.6 \mathrm{GeV}$, so having one more energy point around $15 \mathrm{GeV}$ would allow to confirm the trends happening between 11.5 and $19.6 \mathrm{GeV}$. 


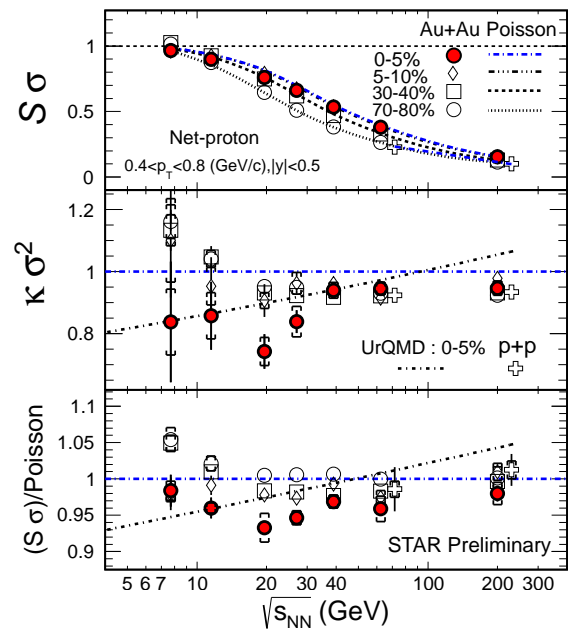

Table 1: The collider mode $\sqrt{s_{N N}}$, fixed target mode $\sqrt{s_{N N}}$, and corresponding $\mu_{B}$ values. The $\mu_{B}$ values are listed for the central collisions corresponding to fixed target $\sqrt{s_{N N}}[10]$.

\begin{tabular}{c|c|c}
\hline $\begin{array}{c}\text { Collider mode } \\
\sqrt{s_{N N}}(\mathrm{GeV})\end{array}$ & $\begin{array}{c}\text { Fixed-target mode } \\
\sqrt{s_{N N}}(\mathrm{GeV})\end{array}$ & $\begin{array}{c}\mu_{B} \\
(\mathrm{MeV})\end{array}$ \\
\hline 19.6 & 4.5 & 585 \\
15 & 4.0 & 625 \\
11.5 & 3.5 & 670 \\
7.7 & 3.0 & 720 \\
5 & 2.5 & 775 \\
\hline
\end{tabular}

Figure 7: (Color online) $\kappa \sigma^{2}, S \sigma$ and $S \sigma$ values normalized by the Poisson expectation, as a function of collision energy. Vertical bars are statistical and caps are systematic errors.

In view of these, STAR has proposed a BES Phase-II program. For this STAR has requested an electron cooling device at RHIC to increase the luminosity for collisions below $\sqrt{s_{N N}}=20$ $\mathrm{GeV}$. Simulation results indicate that with electron cooling, the luminosity could be increased by a factor of about 3-5 at $7.7 \mathrm{GeV}$ and about 10 around $20 \mathrm{GeV}$ [30]. An additional improvement in luminosity may be possible by operating with longer bunches at the space-charge limit in the collider [31]. So overall improvement in luminosity could be about 10-fold. The luminosity improvement will not only allow the precision measurements of the above important observables but will also be helpful in the measurements of rare probes such as dilepton production and hypertriton measurements [32]. To maximize the use of collisions provided at STAR for the BES program, we have proposed to run STAR in "fixed-target mode". The main motivation for this proposal is to extend the $\mu_{B}$ coverage currently from $400 \mathrm{MeV}$ to about $800 \mathrm{MeV}$ so as to cover a large portion of the phase diagram. For this, we plan to install a fixed Au target in the beam pipe to perform the $\mathrm{Au}$ (beam)-Au(target) collisions. The data taking can be done concurrently during the normal RHIC running. This proposal will not affect the normal RHIC operations. Table 1 lists the proposed collision energies, corresponding fixed target center-of-mass energies, and baryon chemical potential values.

\section{Summary}

We have presented several interesting results from the STAR BES Phase-I program. Currently, STAR covers a large range of $\mu_{B}(20-400 \mathrm{MeV})$ in the phase diagram. We have observed a centrality dependence of freeze-out parameters at lower energies. We have looked at various observables for the search of first order phase transition, "turn-off" of the QGP signatures, and search of a QCD critical point. The observables such as $v_{1}$ slope, $R_{\mathrm{CP}}$ and $v_{2}$ of PID hadrons, and higher moments of net-proton distributions, show some dramatic changes for the energy range 
$\sqrt{s_{N N}}<20 \mathrm{GeV}$. We have observed that proton $v_{1}$ slope changes sign between 7.7 and $11.5 \mathrm{GeV}$. The net-proton $v_{1}$ slope changes sign twice as a function of beam energy. We have observed a difference in $v_{2}$ of particles and corresponding anti-particles which increases with decreasing collision energy. The $\phi$-meson $v_{2}$ deviates from that of other particles, the charge separation signal vanishes, and the $R_{\mathrm{CP}}$ of $K_{S}^{0}$ is greater than unity for beam energy of $11.5 \mathrm{GeV}$ and below. The moment products have values below the Poisson expectation for $0-5 \%$ central $\mathrm{Au}+\mathrm{Au}$ collisions. For peripheral collisions, they are above unity at $\sqrt{s_{N N}}<19.6 \mathrm{GeV}$. An electron cooling device at RHIC will significantly increase luminosity at beam energies below $20 \mathrm{GeV}$ allowing the precision measurements for several important observables for phase structure studies. In addition, we propose to run STAR in fixed target mode which will extend the $\mu_{B}$ coverage from STAR up to $800 \mathrm{MeV}$ in the phase diagram.

We acknowledge the support from DOE for this research.

\section{References}

[1] J. Adams et al. (STAR Collaboration), Nucl. Phys. A 757, 28 (2005).

[2] Y. Aoki et al., Nature 443 675, (2006).

[3] M. M. Aggarwal et al. (STAR Collaboration), arXiv:1007.2613 L. Kumar (for STAR Collaboration), Nucl. Phys. A 862, 125 (2011); B. Mohanty, Nucl. Phys. A 830, 899C (2009).

[4] B. I. Abelev et al. (STAR Collaboration), Phys. Rev. C 81, 024911 (2010).

[5] M. Anderson et al., NIM A 499, 659 (2003); W. Llope (for STAR Collaboration), NIM A 661, S110 (2012).

[6] L. Kumar (for STAR Collaboration), arXiv:1201.4203 2012), J. Phys. G: Nucl. Part. Phys. 38, 124145 (2011); X. Zhu (for STAR Collaboration), Acta Phys. Polon. B Proc. Supp. 5, 213 (2012).

[7] J. Adams et al. (STAR Collaboration), Nucl. Phys. A 757, 102 (2005); J. Cleymans et al., Comp. Phys. Comm. 180, 84 (2009)

[8] S. Das (for STAR Collaboration), these proceedings.

[9] A. Andronic et al., Nucl. Phys. A 834, 237 (2010).

[10] J. Cleymans et al., Phys. Rev. C 73, 034905 (2006).

[11] E. Schnedermann et al., Phys. Rev. C 48, 2462 (1993).

[12] J. Brachmann et al., Phys. Rev. C 61, 024909 (2000); L. P. Csernai et al., Phys. Lett. B 458, 454 (1999); H. Stocker, Nucl. Phys. A 750, 121 (2005)

[13] Y. Pandit (for STAR Collaboration), these proceedings.

[14] J. Adams et al. (STAR Collaboration), Phys. Rev. Lett. 95, 122301 (2005).

[15] S. Shi (for STAR Collaboration), these proceedings.

[16] J. Dunlop et al., Phys. Rev. C 84, 044914 (2011); J. Xu et al., Phys. Rev. C 85, 041901 (2012).

[17] B. Mohanty and N. Xu, J. Phys. G 36, 064022 (2009).

[18] B. I. Abelev et al. (STAR Collaboration), Phys. Rev. Lett. 103, 251601 (2009).

[19] K. Fukushima et al., Phys. Rev. D 78, 074033 (2008).

[20] G. Wang (for STAR Collaboration), these proceedings.

[21] B. Abelev et al. (ALICE Collaboration), arXiv:1207.0900

[22] M. A. C. Lamont (for the STAR Collaboration), J. Phys. Conf. Ser. 50, 192 (2006).

[23] X. Zhang (for STAR Collaboration), these proceedings.

[24] E. Sangaline (for STAR Collaboration), these proceedings.

[25] M. A. Stephanov, Phys. Rev. Lett. 102, 032301 (2009); M. A. Stephanov, Phys. Rev. Lett. 107, 052301 (2011).

[26] S. Gupta et al., Science 332, 1525 (2011); F. Karsch and K. Redlich, Phys. Lett. B 695, 136 (2011).

[27] X. Luo (for STAR Collaboration), these proceedings.

[28] M. Bleicher et al., J. Phys. G: Nucl. Part. Phys. 25, 1859 (1999).

[29] D. McDonald (for STAR Collaboration), these proceedings; P. Tribedy (for STAR Collaboration), these proceedings.

[30] A. Fedotov, and W. Fischer, Private communications, 2012.

[31] A. Fedotov and M. Blaskiewicz, BNL CAD Tech Note: C-A/AP/449 (February 10, 2012).

[32] B. Huang (for STAR Collaboration), these proceedings; Y. Zhu (for STAR Collaboration), these proceedings. 